\title{
OBJECT-ORIENTED CHANGE DETECTION BASED ON WEIGHTED POLARIMETRIC SCATTERING DIFFERENCE ON POLSAR IMAGES
}

\author{
Xuejing Shi ${ }^{\mathrm{a}}$, Lijun $\mathrm{Lu}^{\mathrm{a}}$, Shucheng Yang ${ }^{\mathrm{a}}$, Guoman Huang ${ }^{\mathrm{a}}$, Zheng Zhao* \\ ${ }^{a}$ Chinease Academy of Surveying \&Mapping, Beijing, China-SXJ_shixuejing @ 163.com
}

KEY WORDS: Object-oriented change detection, Polarimetric scattering difference, Polarimetric synthetic aperture radar

\begin{abstract}
:
For wide application of change detection with SAR imagery, current processing technologies and methods are mostly based on pixels. It is difficult for pixel-based technologies to utilize spatial characteristics of images and topological relations of objects. Object-oriented technology takes objects as processing unit, which takes advantage of the shape and texture information of image. It can greatly improve the efficiency and reliability of change detection. Recently, with the development of polarimetric synthetic aperture radar (PolSAR), more backscattering features on different polarization state can be available for usage of object-oriented change detection study. In this paper, the object-oriented strategy will be employed. Considering the fact that the different target or target's state behaves different backscattering characteristics dependent on polarization state, an object-oriented change detection method that based on weighted polarimetric scattering difference of PolSAR images is proposed. The method operates on the objects generated by generalized statistical region merging (GSRM) segmentation processing. The merit of GSRM method is that image segmentation is executed on polarimetric coherence matrix, which takes full advantages of polarimetric backscattering features. And then, the measurement of polarimetric scattering difference is constructed by combining the correlation of covariance matrix and the difference of scattering power. Through analysing the effects of the covariance matrix correlation and the scattering echo power difference on the polarimetric scattering difference, the weighted method is used to balance the influences caused by the two parts, so that more reasonable weights can be chosen to decrease the false alarm rate. The effectiveness of the algorithm that proposed in this letter is tested by detection of the growth of crops with two different temporal radarsat-2 fully PolSAR data. First, objects are produced by GSRM algorithm based on the coherent matrix in the pre-processing. Then, the corresponding patches are extracted in two temporal images to measure the differences of objects. To detect changes of patches, a difference map is created by means of weighted polarization scattering difference. Finally, the result of change detection can be obtained by threshold determining. The experiments show that this approach is feasible and effective, and a reasonable choice of weights can improve the detection accuracy significantly.
\end{abstract}

\section{INTRODUCTION}

In recent years, PolSAR (polarimetric Synthetic Aperture Radar) as the more advanced imaging radar can obtain the fully polarimetric information of targets. It has unique advantages compared to the single polarimetric SAR in analysing and extracting the polarimetric scattering characteristics of ground objects. Due to the complex nature of manmade structures, PolSAR analysis becomes highly challenging (Bhogendra Mishra, 2013). At present, many scholars have studied the SAR change detection technology, but most methods are based on the pixel. With pixel-based change detection, comparison is applied on the pixel level (X. Chen, 2008). It is easy to be affected by speckle noise. The pixel-based technologies have been difficult to meet the application requirements since the complexity of SAR imaging. Object-oriented technology takes objects as processing unit can solve the bottleneck effectively. The objectoriented analysis can obtain a variety of additional spatial and textural information (Sun Xiaoxia, 2013). It is important for improving the accuracy of change detection. With the aim being to improve the efficiency and reliability of change detection, the polarimetric scattering information is used to measure the similarity of patches and distinguish the changes of images. In this paper, it will combine the object-oriented technology and fully PolSAR information to detect the changes of crop growth in different periods.

\section{METHODS}

The technology that object-oriented change detection based on the polarimetric scattering characteristics is measuring the diversity of corresponding patches in different images. For multi-temporal and quad-PolSAR images, the backscattering properties will change with the objects changing. Here, $C_{x}$ and $C_{y}$ represent the covariance matrices of two goals in image x and image y respectively, $P_{x}$ and $P_{y}$ are expressed as their echo power. The measurement of polarimetric scattering difference is constructed by combining the correlation of covariance matrix and the difference of scattering power.

$$
D\left(C_{x}, C_{y}\right)=\left(1-\left\langle\frac{C_{x}}{\left\|C_{x}\right\|_{F}} \cdot \frac{C_{y}}{\left\|C_{y}\right\|_{F}}\right\rangle\right)+\left(1-\frac{2}{P_{x} / P_{y}+P_{y} / \mathrm{P}_{x}}\right)
$$

Where $\left\|C_{x}\right\|_{F}$ and $\left\|C_{y}\right\|_{F}$ are Frobenus norm of the polarimetric covariance matrices $C_{x}$ and $C_{y},\langle\cdot\rangle$ indicates the inner product of matrices. $P_{x}$ and $P_{y}$ are the scattered power of image $\mathrm{x}$ and image $\mathrm{y}$. The scattered power is defined as:

$$
P=\left|S_{H H}\right|^{2}+2\left|S_{H V}\right|^{2}+\left|S_{V V}\right|^{2}
$$

The greater of scattering difference that corresponding patches in different temporal images, the more likely to change.

Because the measurement of polarimetric scattering difference is constructed by combining the correlation of covariance matrix (Muhtar Qong, 2004) and the difference of

\footnotetext{
* Corresponding author
} 
scattering power, these two parts are the factors that influence the polarimetric scattering difference. Theoretically, the same objects in different images show the uniform scattering and power properties under the same imaging condition. When the same location of surface targets in different temporal image have changed, that means the scattering and power characteristics of the target have changed accordingly. However, the extent of changes is not the same, the effect of scattering characteristics may dominate or the effect of echo power characteristics may dominate (Han Ping, 2013). For scattering polarization diversity these two parts influence on the overall difference degree should be considered. To solve this problem, the polarization scattering differential are weighted on the formula (1). It can be expressed as: $D\left(C_{x}, C_{y}\right)=a \cdot D_{C}+b \cdot D_{P}$

$$
=a \cdot\left(1-\left\langle\frac{C_{x}}{\left\|C_{x}\right\|_{F}} \cdot \frac{C_{y}}{\left\|C_{y}\right\|_{F}}\right\rangle\right)+b \cdot\left(1-\frac{2}{P_{x} / P_{y}+P_{y} / \mathrm{P}_{x}}\right)
$$

Where $\mathrm{a}$ and $\mathrm{b}$ are so-called weighted coefficient, which determine the two parts of factors on the contribution degree of polarization scattering difference.

\section{DATA AND PREPROCESSING}

In this part, the two Radarsat-2 polarimetric SAR data of Gen River experimentation area is used to conduct experiment, which were acquired in August 3, 2013 and August 27, 2013. The land use types of plain mostly are farmland, and the images of experimentation area are shown in figure 1.The main area of the field investigation is Shangkuli farm, of which farmlands are of regular shape, large area, and terrain topography is flat. The main crops are rape, wheat, barley, U.S.wheat, and weeds. Although a few of features can be utilized, the different states of the ground caused by terrain scattering model are more diverse, which are suitable for change detection. The farm types (experimentation area) are shown in figure 2.

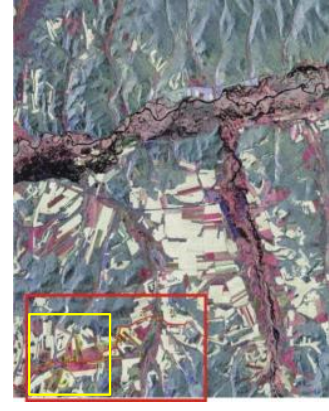

(a)

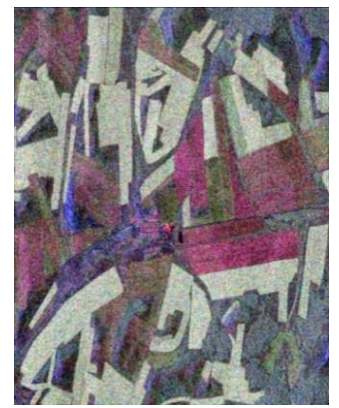

(b)
Figure 1. Radarsat-2 images of Gen River: (a) Pauli synthetic images, (b) Experimental area (the yellow frame)

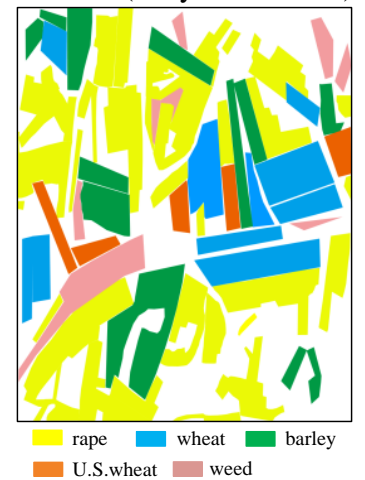

Figure 2. The first team of Shangkuli farm species type
The data need to be pre-processed before detecting changes, including speckle noise suppression and image registration. For its coherent imaging mechanism, SAR images are inevitably corrupted by speckle noises (Lee, 2009). It is difficult to recognize terrain target and classify land cover. Therefore, it is necessary and useful to suppress speckle noises In addition, due to the various sources of the image, change detection has to establish the one-to-one relationship between the pixels in multiple images. The geometrical co-registration is very important in a change detection application where two images are compared on a pixel-by-pixel basis (Allan A. Nielsen, 2007). Figure 3 shows the polarization SAR images after pre-processing.
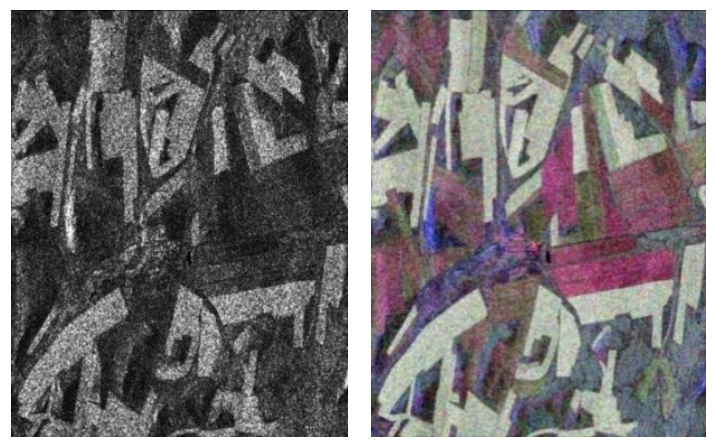

(a)
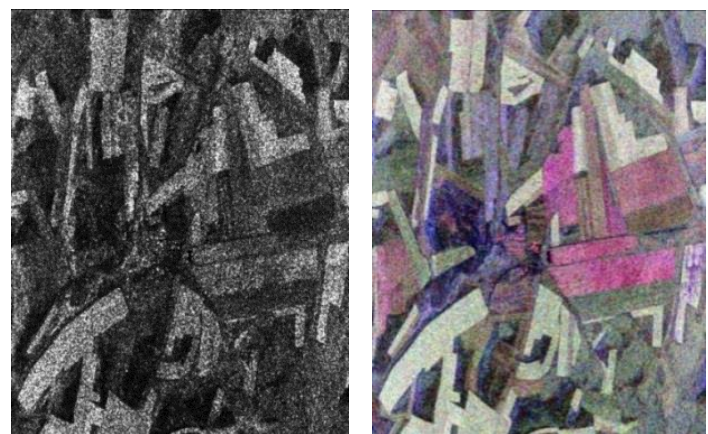

(b)

Figure 3. HH polarization and Pauli synthesis of SAR images after preprocessing, (a) Image 1, (b) Image 2

The targets response is usually anisotropic of SAR image. In order to facilitate comparison between multi-temporal SAR images with corresponding regional changes, efficient image segmentation method is needed to object-based change detection. It is a process of partitioning an image into homogenous groups that each region is homogenous but the union of no two adjacent regions is homogenous (V. Dey, 2010; Craves R.G, 1994). Image segmentation is the core of object-oriented technology. The method operates on the objects generated by generalized statistical region merging (GSRM) segmentation processing (Lang F, 2014). GSRM segmentation method is executed on polarimetric coherence matrix, which takes full advantages of polarimetric backscattering features. Objects are produced by GSRM algorithm based on the coherent matrix in the pre-processing. Figure 4 shows the vector layer overlapped original image after image segmentation. 


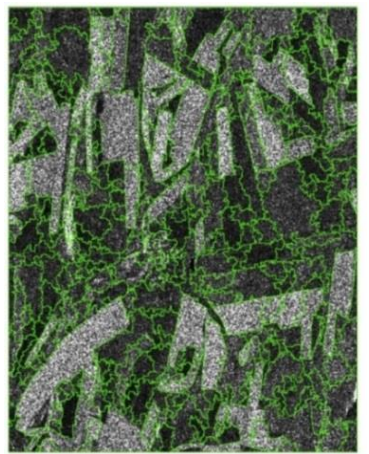

(a)

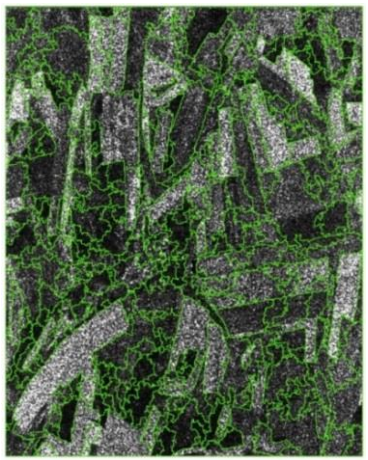

(b)
Figure 4. Results of PolSAR images segmentation, (a) Image 1, (b) Image 2

\section{EXPERIMENT AND ANALYSIS}

The crops changed as the different growth period, which leads to the change of scattering characteristics. To test the change detection method that based on polarimetric scattering difference, the corresponding patches are first extracted to measure the diversity by formula (1). It can produce a difference map. Then, the method of circulating segmentation is used to judge the difference values of patches in different images. The threshold is determined to distinguish changes in the patches to generate change detection results of binary image. The fundamental is setting the initial threshold $T_{0}$, the difference map will be discriminated pixel by pixel with initial threshold $T_{0}$. If the pixel is greater than $T_{0}$, it will be divided into the image block $I_{l}$, otherwise, other pixels will be divided into the image block $I_{2}$. The mean value of block $I_{1}$ and block $I_{2}$ are calculated respectively, and the threshold $T^{\prime}$ can be recalculated by the function $T^{\prime}=\left(\mu_{1}+\mu_{2}\right) / 2$, the absolute value of the difference between the initial threshold $T_{0}$ and $T^{\prime}$ ' will be judged, the best threshold will be determined by the initial value of threshold after several iterations.

The mean of objects' polarimetric scattering differences 0.582 is used in the experiment for initial classification of patches spots. The average of unchanged classes and changed classes are calculated as $\mu 1$ and $\mu 2$ respectively. Then, $\mathrm{T}$ '= $(\mu 1+\mu 2) / 2=2.72$. The parameter $\Delta \mathrm{T}$ can be computed by the function $\Delta \mathrm{T}=\left|\mathrm{T}^{\prime}-\mathrm{T}\right|$. Compared with $\Delta \mathrm{T}$ and constant 0.01 and repeat the above steps, the successive iteration until the parameter $\Delta \mathrm{T}$ is less than 0.01 (0.01 is an empirical value, it can set according to the actual needs). Until the fourth iteration, $\mathrm{T}^{\prime}=0.752$ and $\Delta \mathrm{T}=0.007<0.01$, the iteration is stopped at 0.752 and it is the optimal threshold. The relationship of size between polarimetric scattering difference of patches and threshold are judged in difference map (Meng Liu, 2012). If polarimetric scattering difference is greater than the threshold, the patches are divided into the changed patches. While polarimetric scattering differences is less than the threshold, the patches are divided into unchanged patches. Figure 6 shows the change detection results based on the difference of polarimetric scattering. Where (a) and (b) show the image 1 and image 2, (c) is the difference map and (d) is the binary image of change detection results. The black means changed patches and the white means unchanged patches.

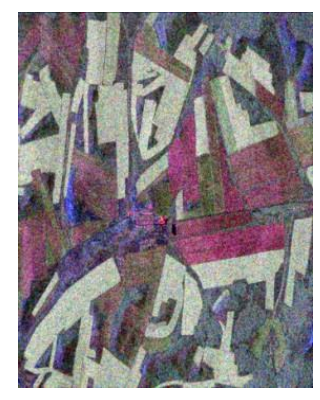

(a)

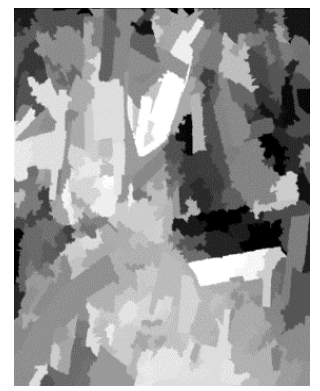

(c)

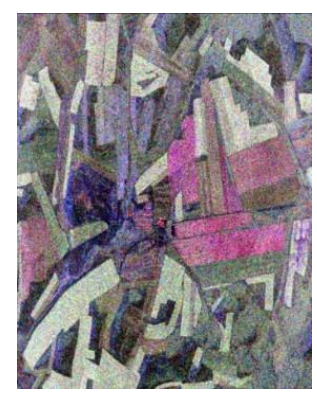

(b)

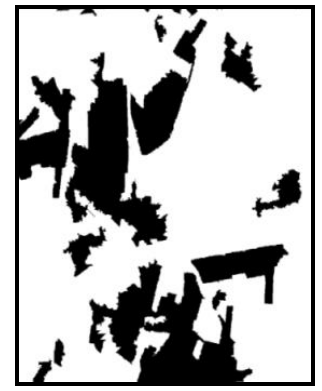

(d)
Figure 5. The change detection results based on the difference of polarimetric scattering, (a) image 1, (b) image 2, (c) difference map, (d) the results of change detection

Fifty patches are chosen as samples random to evaluate the accuracy of the change detection results. In the extracted 50 samples, combined with the results of field location-survey, there are twenty patches have actually changed and that are detected as changed objects. The number of objects that have not changed and not be detected as changed patches is 23 . The number of objects that have actually changed but not be detected for changed patch is 2 , and there are five patches that have not changed but that are detected for the changed patches. Table 1 show that the accuracy evaluation of the detection performance of the change.

\begin{tabular}{|c|c|c|c|c|}
\hline $\begin{array}{c}\text { Evaluation } \\
\text { index }\end{array}$ & $\begin{array}{c}\text { Accuracy } \\
1 \%\end{array}$ & $\begin{array}{c}\text { Omission } \\
\text { factor } \\
1 \%\end{array}$ & $\begin{array}{c}\text { False } \\
\text { alarm } \\
\text { rate } \\
1 \%\end{array}$ & $\begin{array}{c}\text { General } \\
\text { error } \\
\text { rate } \\
1 \%\end{array}$ \\
\hline $\begin{array}{c}\text { unweighted } \\
\text { method }\end{array}$ & 86.0 & 9.0 & 17.8 & 14.0 \\
\hline
\end{tabular}

Table 1. The precision evaluation of change detection results based on the polarimetric scattering difference

The effective use of topological relationship and neighbourhood features between images is one of the advantages for object-oriented change detection. It is convenient for the later qualitative analysis. Ten typical samples that cover five planting types of the field annotation are selected to analyse the change detection results. The ten samples include changed patches and unchanged patches. Figure 6 shows that the samples distribution of image segmentation and the farm type map. Sample 1 and 5 are barley, the sample 2, 9 and 10 are the rape, sample 3 and 7 are wheat, sample 6 is U.S. wheat, and sample 4 and 8 are weeds. The table 2 shows the polarization scattering characteristics of the parameters, including the correlation of covariance matrix 
and the scattering difference of two images. Figure 7 shows the distribution of the polarimetric scatter difference.

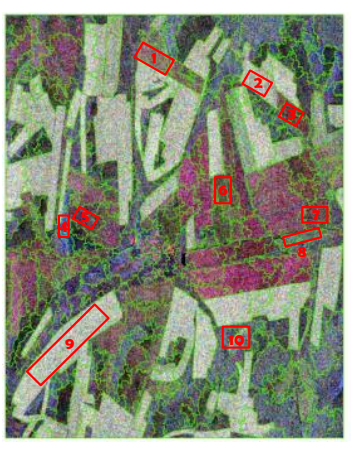

(a)
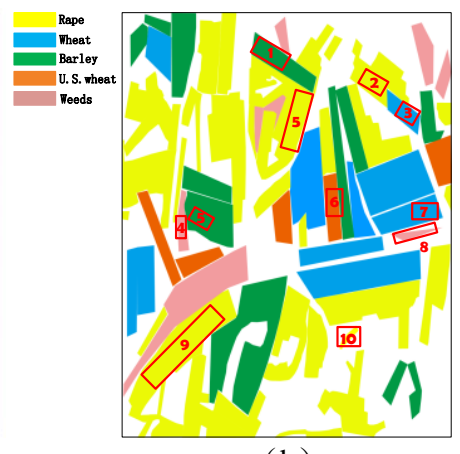

(b)
Figure 6. The samples distribution, (a) The image after segmentation, (b) The first team of Shangkuli farm species type

\begin{tabular}{|c|c|c|c|}
\hline Samples & $\begin{array}{c}\text { Polarimetric } \\
\text { scattering } \\
\text { differences }\end{array}$ & $\begin{array}{c}\text { covariance } \\
\text { matrix } \\
\text { correlation }\end{array}$ & $\begin{array}{c}\text { echo } \\
\text { power } \\
\text { differences }\end{array}$ \\
\hline 1 & 0.437 & 0.014 & 0.424 \\
\hline 2 & 0.758 & 0.004 & 0.754 \\
\hline 3 & 0.279 & 0.072 & 0.207 \\
\hline 4 & 0.943 & 0.002 & 0.942 \\
\hline 5 & 0.838 & 0.042 & 0.796 \\
\hline 6 & 0.317 & 0.014 & 0.303 \\
\hline 7 & 0.803 & 0.124 & 0.679 \\
\hline 8 & 0.768 & 0.006 & 0.762 \\
\hline 9 & 0.602 & 0.002 & 0.600 \\
\hline 10 & 0.771 & 0.004 & 0.768 \\
\hline
\end{tabular}

Table 2. Polarimetric scattering parameters

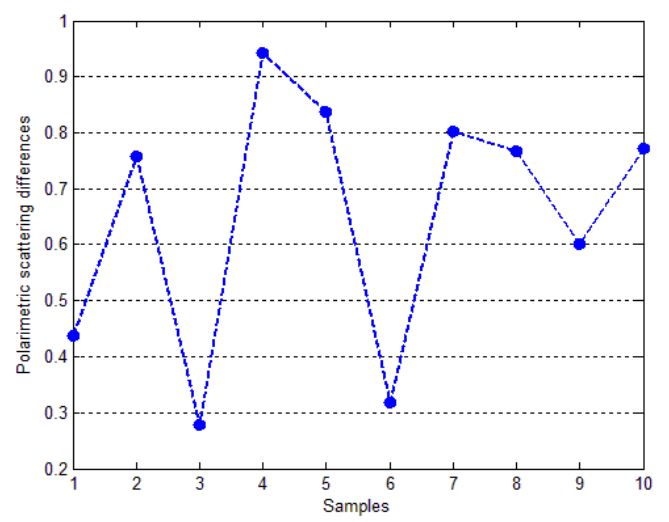

Figure 7. The differences distribution of polarimetric scattering

The distribution of polarimetric scattering difference is utilized to analyse the change of objects. The automatic threshold is 0.752 in the process of change detection. The patches are detected as changed objects are samples 2, 4, 5, 7, 8,10 , which combined with the change detection results (Figure $6(\mathrm{~d})$ ) and the polarimetric scattering diversity (Figure
8 (a)). However, actual changes in the patches for 2, 4, 5, 7, 8 that combined with the field survey results, sample 10 does not change obviously. But it is detected as a changed object in this method, and its covariance matrix of correlation is 0.004 and echo power difference is 0.768 . The high false alarm may be caused by the too strong influence of echo power.

The correlation of covariance matrix and echo power difference have an impact on polarimetric scattering difference. To estimate the extent of this two parts impact of the polarimetric scattering difference, the following discussion only consider one of the two factors. If correlation of covariance matrix is only considered and the echo power difference is ignored, the experiment found that miss rate is very high when $a=1, b=0$. However, when $a=0, b=1$, the echo power difference is only considered and the correlation of covariance matrix is ignored, it was found that the false alarm rate is very large. As shown in figure 8 , that are the difference images and results of change detection.

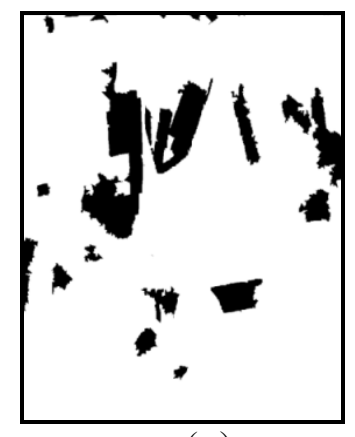

(a)

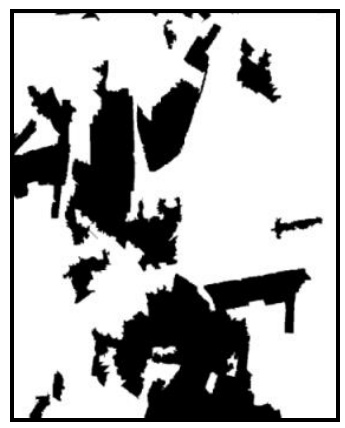

(b)
Figure 8 . The results of change detection, (a) $a=1, b=0$, (b) $a=0$, $\mathrm{b}=1$

Experiments show that it is important for the polarimetric scattering difference that correlation of covariance matrix and echo power difference, and both of them are indispensable. So in order to employ polarimetric scattering information of full polarimetric SAR data in change detection effectively, correlation of covariance matrix and echo power differences will be combined with joint weighted. And the better results will be obtained through assigning weights. In fact, the effect of echo power of image will far more than the effect of scattering characteristics of image. For the original method that unweighted, since the echo power has impact on the whole scattering diversity too strongly, the change detection results have high false alarm rate. The weight should be assigned to balance the influences caused by the two parts so as to decrease the false alarm rate. Weighted coefficient a and b should be divided according to the size of this two parts' difference degree. So as to restrain the effect on the echo power difference and improve the scattering contribution to the degree of dissimilarity, weighted coefficients are selected in $\mathrm{a}>0.5, \mathrm{~b}<0.5$.

The weights of four groups are chosen in this experiment. The changes of patches will be measure based on weighted polarimetric scattering difference. Different weights allocation will cause the contribution of covariance correlation matrix and scattering power difference to polarimetric scattering difference, which will lead to change threshold. The results of detection will be changed with the threshold changed. Table 3 shows the weight distribution and the size of the corresponding threshold. And figure 9 is the binary image that change detection results of different weight distribution. 


\begin{tabular}{|c|c|c|}
\hline $\mathrm{a}$ & $\mathrm{b}$ & Threshold \\
\hline 0.6 & 0.4 & 0.304 \\
\hline 0.7 & 0.3 & 0.232 \\
\hline 0.8 & 0.2 & 0.162 \\
\hline 0.9 & 0.1 & 0.091 \\
\hline
\end{tabular}

Table 3. Weight distribution and the size of the corresponding threshold

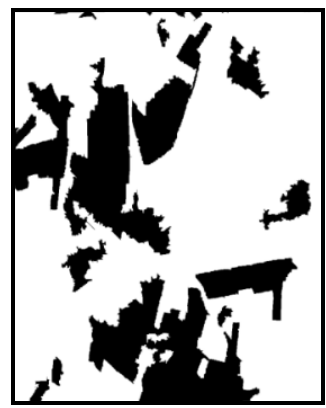

(a)

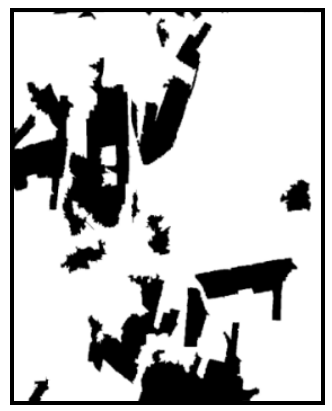

(c)

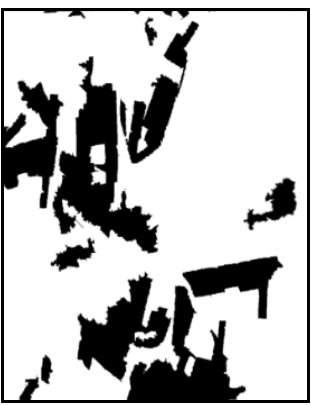

(b)

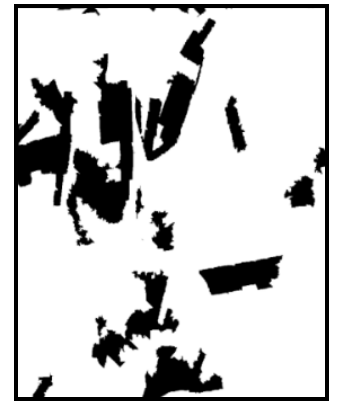

(d)
Figure 9. Change detection results of different weight distribution, (a) $a=0.6, b=0.4$, (b) $a=0.7, b=0.3$, (c) $a=0.8, b=0.2$, (d) $a=0.9, b=0.1$

As shown in figure 9. When $a=0.8, b=0.2$ and $a=0.9, b=0.1$ the miss rate is higher, this is caused by excessive suppression of echo power difference. The detection results of primal unweighted method, when $a=0.6, b=0.4$ and when $a=0.7, b=0.3$ are compared to analysis the change detection results, the five kinds of significant change areas are noted.

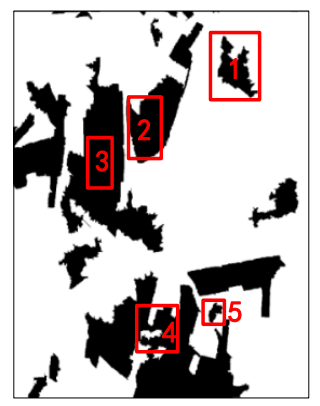

(a)

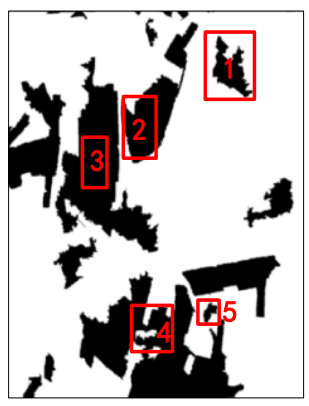

(b)

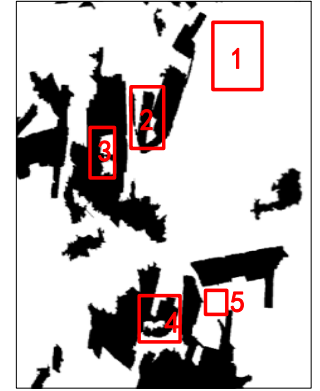

(c)

Figure 10. The comparison of change detection results, (a) original method, (b) $a=0.6, b=0.4$, (c) $a=0.7, b=0.3$

Since the excessive suppression of echo power difference, region 1 actually changed according to the results of field annotation, and in figure (c) it did not detected as changed object. Region 2, region 3、 region 4 and 5 all are excessive detection in figure 10 (a) and (b). For example, region 2 actually changed map patches as shown in Figure 11, the red area is unchanged in the patches, but in figure 10 (a) and (b), this patches are detected as changed objects. Resulting in this phenomenon is the original method and the method that weights according to $\mathrm{a}=0.6, \mathrm{~b}=0.4$ allocation are influenced by echo power, and this weights allocation did not balance the influence of echo power difference on the overall diversity. After comparison, when the weights are assigned by $a=0.6, b=0.4$, that don't restrain echo power difference on the influence of polarimetric scattering dissimilarity. The change detection results still have high false alarm rate. However, when the weights are assigned by $a=0.8, b=0.2$ and by $a=0.9 b=0.1$, because the excessive suppression of echo power difference result in the missed phenomenon is more serious. Comprehensive the above situation, the detection results is the optimal when $\mathrm{a}=0.7, \mathrm{~b}=0.3$.

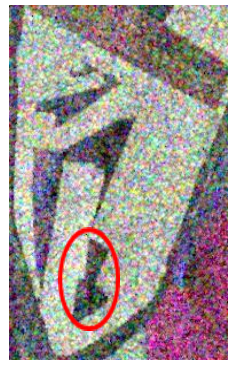

(a)

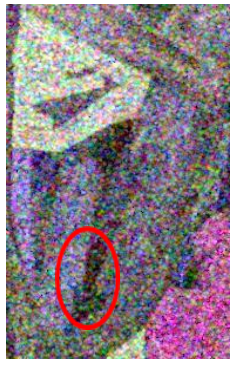

(b)

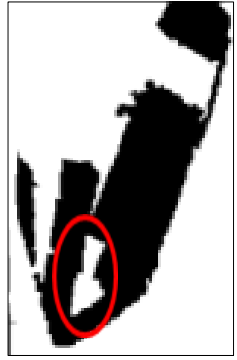

(c)
Figure 11. The detail drawings of area 2 and detection result, (a) image 1 , (b) image 2 , (c) the patch of change detection

Fifty patches are selected as samples randomly to assess the detection performance. Table 5.10 is the precision evaluation of different weights allocation change detection results. Table 5 shows that the correct rate increases to $94 \%$ when authority value $a=0.7, b=0.3$, and the missing rate and false alarm rate are reduced largely. Thereby the overall accuracy is improved and the total error rate is decreased. From the perspective of qualitative and quantitative comprehensive analysis, change detection result is optimal when $a=0.7, b=0.3$, the weight allocation method can overcome the original method based on polarization difference diversity for the detection of a change with the higher false alarm rate in a large extent, the desired results have been achieved. 


\begin{tabular}{|c|c|c|c|c|}
\hline $\begin{array}{c}\text { Evaluation } \\
\text { index }\end{array}$ & $\begin{array}{c}\text { Accuracy } \\
/ \%\end{array}$ & $\begin{array}{c}\text { Omission } \\
\text { factor } \\
1 \%\end{array}$ & $\begin{array}{c}\text { False } \\
\text { alarm } \\
\text { rate } \\
1 \%\end{array}$ & $\begin{array}{c}\text { General } \\
\text { error } \\
\text { rate } \\
1 \%\end{array}$ \\
\hline $\begin{array}{c}\text { original } \\
\text { method }\end{array}$ & 86.0 & 9.0 & 17.8 & 14.0 \\
\hline $\mathrm{a}=0.6, \mathrm{~b}=0.4$ & 86.0 & 9.0 & 17.8 & 14.0 \\
\hline $\mathrm{a}=0.7, \mathrm{~b}=0.3$ & 94.0 & 4.5 & 7.1 & 6.0 \\
\hline $\mathrm{a}=0.8, \mathrm{~b}=0.2$ & 88.0 & 18.2 & 7.1 & 12.0 \\
\hline $\mathrm{a}=0.9, \mathrm{~b}=0.1$ & 86.0 & 22.7 & 7.1 & 14.0 \\
\hline
\end{tabular}

Table 5. The precision evaluation of different weights allocation change detection results

\section{CONCLUSIONS}

First, the unweighted polarimetric scattering difference is used to experiment. And it turns out the false alarm rate is overly large leads to a decline in the overall accuracy. The difference between the covariance matrix of the two images and the difference of the scattering power both are the factors of the polarization scatter difference. Through the experimental analysis, the difference of echo power is much higher than the correlation of the covariance matrix of the polarization. However, the cause of this result is that the difference of echo power has a great influence on the polarization scatter difference, which has affected the results of the change detection. To solve this problem, this paper proposes a change detection method that joint weighted polarization scattering difference. It overcomes the problem of serious false alarm arised in the unweighted method. The experimental results show that the false alarm rate of the two parts is obviously decreased, and the accuracy of the change detection is improved. The results of this experiment show that a reasonable choice of weights can improve the detection accuracy significantly.

\section{ACKNOWLEDGEMENTS}

Acknowledgements of support by Special Fund by Surveying \& Mapping and Geoinformation Research in the Public Interest (201412010).

\section{REFERENCES}

Allan A. Nielsen, Henning Skriver, Knut Conradsen, 2007. Complex Wishart Distribution Based Analysis of Polarimetric Synthetic Aperture Radar Data. International Workshop on the Analysis of Multi-temporal Remote Sensing Images, pp. 1-6.

Bhogendra Mishra, Junichi Susaki, 2013. Unsupervised Change Detection in an Urban Environment Using Multitemporal PolSAR Images. IEEE Proceedings on the JURSE 2013, pp. 45-48.

Craves R.G, Quegan S, 1994. Segmentation based change detection in ERS-1 SAR images. IEEE Transactions on Geoscience and Remote Sensing, 4, pp. 2149-2151.
Han Ping, Cong runmin, 2013. Change Detection Algorithm of Polarimetric SAR Image Based on Joint Weighted Polarization Difference Degree. Journal of signal processing, 29(10), pp. 1390-1397.

Lang F, Yang J, Li D, 2014. Polarimetric SAR Image Segmentation Using Statistical Region Merging. IEEE Geoscience and Remote Sensing Letters, 11(2), pp. 509-513.

Lee, J.S., Pottier, E., 2009. Polarimetric radar imaging from basics to applications. CRC Press, Boca Raton, pp. 53-84.

Sun Xiaoxia, Zhang Jixian, Yan Qin, 2013. Object-oriented change detection of multi-polarization SAR images based on unitemporal image segmentation. IEEE. JURSE 2013, pp. 171173.

Meng Liu, Hong Zhang, Chao Wang, et al, 2012. PolSAR Change Detection for Specific Land Cover Type by Testing Equality of Two PollnSAR Coherency Matrixes. 2012 International Conference on computer Vision in Remote Sensing (CVRS), pp. 371-376.

Muhtar Qong, 2004. Polarization State Conformation and Its Application to Change Detection in Polarimetric SAR Data. IEEE Transactions on Geoscience and Remote Sensing, 1(40), pp. 304-308.

V. Dey, Y. Zhang, M. Zhong, 2010. A review on image segmentation techniques with remote sensing perspective. ISPRS TC VII Symposium - 100 Years ISPRS, Vol. XXXVIII, pp. 31-42.

X. Chen, F. Wu, C. Wang, 2008. Change Detection with MultiPolarization SAR Imagery. IEEE Transactions on Geoscience and Remote Sensing Symposium, 4, pp. 659-662. 